\title{
CORRECTION
}

Open Access

\section{Correction to: First description of Echinococcus ortleppi infection in China}

\author{
Yunliang Shi ${ }^{1}$, Xiaoling Wan ${ }^{1}$, Ziyue Wang ${ }^{2}$, Jun $\mathrm{Li}^{1}$, Zhihua Jiang ${ }^{1}$ and Yichao Yang ${ }^{1 *}$
}

Correction to: Parasites Vectors (2019) 12:398 https://doi.org/10.1186/s13071-019-3653-y

Following publication of the original article [1], the authors reported an error in Table 2.

Namely, in the 'America' section of the table the species 'Crested Porcupine' is matched with the country 'Brazil'.
However, it should be matched with 'Bosnia and Herzegovina' (and so be placed in the 'Europe' section).

The corrected Table 2 is given in this erratum.

*Correspondence: 531174868@qq.com

${ }^{1}$ Institute of Parasitic Disease Prevention and Control, Guangxi Zhuang

Autonomous Region Center for Disease Control and Prevention,

Nanning 530028, China

Full list of author information is available at the end of the article 
Table 2 Summary of animal infection of Echinococcus ortleppi

\begin{tabular}{|c|c|c|c|c|}
\hline Year & Country & Species & No. infected & Reference \\
\hline \multicolumn{5}{|l|}{ Asia } \\
\hline 2009 & India & Cattle/buffalo/pig & $3 / 2 / 4$ & Pednekar et al. [8] \\
\hline 2009 & Vietnam & Monkey & 1 & Pednekar et al. [8] \\
\hline 2015 & Egypt & Camel & 1 & Amer et al. [11] \\
\hline 2016 & Bhutan & Cattle & 1 & Thapa et al. [33] \\
\hline 2017 & Iran & Camel & 1 & Ebrahimipour et al. [34] \\
\hline \multicolumn{5}{|l|}{ Africa } \\
\hline 2004 & Kenya & Cattle/pig & $2 / 1$ & Dinkel et al. [9] \\
\hline 2010-2012 & South Africa & Cow & 1 & Mogoye et al. [27] \\
\hline 2013 & Sudan & Camel & 1 & Ahmed et al. [35] \\
\hline 2013 & Kenya & Cattle/goat/sheep & $23 / 3 / 2$ & Mbaya et al. [10] \\
\hline 2015 & Ethiopia & Cattle/pig & $5 / 1$ & Tigre et al. [36] \\
\hline 2016 & Kenya & Cattle/goat/camel/sheep & $54 / 3 / 2 / 1$ & Addy et al. [3] \\
\hline 2016 & Zambia & Cattle/pig & $52 / 1$ & Addy et al. [3] \\
\hline 2016 & Namibia & Cattle/oryx & $35 / 3$ & Addy et al. [3] \\
\hline 2016 & Ethiopia & Cattle & 7 & Addy et al. [3] \\
\hline 2016 & Sudan & Cattle/camel & $15 / 1$ & Addy et al. [3] \\
\hline 2018 & Kenya & Dog & 1 & Mulinge et al. [37] \\
\hline \multicolumn{5}{|l|}{ America } \\
\hline 2002 & Argentina & Cattle/dog & $5 / 2$ & Kamenetzky et al. [25] \\
\hline 2012 & Brazil & Cattle & 277 & Balbinotti et al. [38] \\
\hline 2016 & Brazil & Cattle & 7 & Addy et al. [3] \\
\hline 2016 & Brazil & Bovine & 250 & Monteiro et al. [39] \\
\hline 2018 & Chile & Cattle & 2 & Correa et al. [41] \\
\hline \multicolumn{5}{|l|}{ Europe } \\
\hline 2012 & UK & Spotted deer & 1 & Boufana et al. [12] \\
\hline 2014 & France & Cattle & 7 & Grenouillet et al. [29] \\
\hline 2016 & France & Cattle & 7 & Addy et al. [3] \\
\hline 2018 & Bosnia and Herzegovina & Crested porcupine & 1 & Hodzic et al. [40] \\
\hline 2008 & Italy & Cattle & 1 & Casulli et al. [42] \\
\hline
\end{tabular}

\section{Author details}

1 Institute of Parasitic Disease Prevention and Control, Guangxi Zhuang Autonomous Region Center for Disease Control and Prevention, Nanning 530028,

China. ${ }^{2}$ School of Public Health, Guangxi Medical University, Nanning 530021, China.

The original article can be found online at https://doi.org/10.1186/s1307 1-019-3653-y.

\section{Reference}

1. Shi Y, Wan X, Wang Z, Li J, Jiang Z, Yang Y. First description of Echinococcus ortleppi infection in China. Parasites Vectors. 2019;12:398. https://doi. org/10.1186/s13071-019-3653-y.

\section{Publisher's Note}

Springer Nature remains neutral with regard to jurisdictional claims in published maps and institutional affiliations.

Published online: 23 August 2019 